\title{
Optimizing temporary perioperative cardiac pacing
}

Henry M. Spotnitz, MD

From the Department of Surgery, Columbia University College of Physicians and Surgeons, New York, NY.

Supported in part by National Institutes of Health grant R01 HL 48109.

Received for publication March 15, 2004; revisions requested March 22, 2004; accepted for publication March 25, 2004.

Address for reprints: Henry M. Spotnitz, MD, Department of Surgery, Columbia University College of Physicians and Surgeons, $622 \mathrm{~W}$ 168th St, PH 14-103, 14th Floor, New York, NY 10032 (E-mail: hms2@columbia.edu).

J Thorac Cardiovasc Surg 2005;129:5-8

$0022-5223 / \$ 30.00$

Copyright (c) 2005 by The American Association for Thoracic Surgery

doi:10.1016/j.jtcvs.2004.03.033

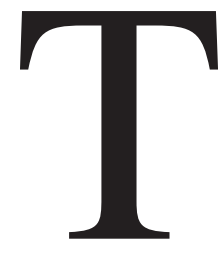

emporary perioperative cardiac pacing (TPCP) is indicated after cardiac surgery for treatment of heart block or sinus bradycardia. ${ }^{1}$ However, TPCP protocols are often arbitrary, lacking routines to maximize cardiac output (CO) and stroke volume (SV). TPCP parameters which can be optimized include heart rate (HR), ventricular pacing site (VPS), and atrioventricular delay (AVD). Also relevant is variable right ventricular-left ventricular delay (RLD) which recently has become available in some permanent pacemakers designed for biventricular pacing (BiVP). Real-time measurement of SV with ultrasonic transit-time aortic flow probes $(\mathrm{UFPs})^{2}$ or arterial pulse contour systems make optimization of CO and SV during changes in TPCP feasible. Dramatic and possibly life-saving benefits of TPCP optimization have been anecdotally reported. Although the theory and practice of TPCP optimization (POPT) will continue to advance, review of current information can lead directly to patient benefits.

\section{Background}

The US Food and Drug Administration (FDA) has approved implantable pulse generators for single-chamber atrial or ventricular (VVI) demand pacing, as well as dual-chamber (DDD) generators that can maintain the physiologic synchrony of atria and ventricles. ${ }^{1}$ Both endocardial (transvenous) and epicardial permanent leads are available. ${ }^{1}$ A typical DDD system uses a single atrial lead and a single ventricular lead. These leads are unipolar or bipolar ( 1 or 2 conductors). Unipolar systems use the body as the indifferent electrode. Transvenous leads for DDD pacing are usually placed in the right atrium (RA) and right ventricle (RV). Epicardial leads are placed externally on the RA, RV, left atrium, or left ventricle (LV). These leads sense intrinsic electrical activity and pace at low energy levels. Implantable pacemakers are adjusted by using programmers that communicate with generators through telemetry. For TPCP after cardiac surgery, temporary external pacemakers are used with removable epicardial wires. Bipolar systems using 2 wires to each chamber avoid common grounds and reduce sensing and pacing artifacts. TPCP parameters are adjusted manually.

Recently, the FDA approved BiVP for congestive heart failure. BiVP is effective in some patients with left ventricular dysfunction and QRS prolongation beyond 120 ms (intraventricular conduction delay [IVCD]). An IVCD and a left ventricular ejection fraction (EF) of less than $35 \%$ often imply loss of synchrony of contraction of the left ventricular free wall and septum. ${ }^{3}$ Clinical trials confirm that endocardial pacing of the RV and LV (through a second pacing lead in a lateral branch of the coronary sinus) narrows the QRS complex and significantly improves objective and subjective measures of cardiac function in selected patients with dilated cardiomyopathy. ${ }^{4,5}$ Small trials indicate that BiVP can improve function while reducing myocardial oxygen consumption $\left(\mathrm{MVO}_{2}\right),{ }^{6}$ that $\mathrm{BiVP}$ is most likely to be effective in patients with very low EF and long IVCD of left bundle branch block configuration, ${ }^{7}$ and that the efficacy of BiVP improves when the left ventricular pacing lead is closest to the most delayed site of left ventricular contraction. ${ }^{3,8}$ Additional studies suggest that endocardial BiVP might be effective in atrial fibrillation and sinus rhythm ${ }^{9}$ and that BiVP reduces the severity of mitral regurgitation. ${ }^{10}$ The efficacy of BiVP might be increased in some patients by incorporating a small (20-80 ms) delay into the pacing algorithm, so that either the LV or RV is stimulated first (DeLurgio DB, personal communication, 2003). The effect of AVD and RLD 
is not consistent in any given patient. RLD optimization more than doubled the improvement in SV obtained with simultaneous RV-LV pacing in an unpublished substudy of the Insync III trial (DeLurgio DB, personal communication).

With epicardial pacing, increasing $\mathrm{HR}$ can increase $\mathrm{CO}$, but the energy cost is also increased. ${ }^{11}$ Compared with VVI, DDD epicardial pacing improves $\mathrm{CO}$ at any given HR. ${ }^{12}$ AVD has been shown to affect $\mathrm{CO}$; this is patient specific over a range of 100 to $225 \mathrm{~ms} .{ }^{13}$ The variable effect of AVD on $\mathrm{CO}$ is related in part to variable conduction times across the atria and ventricles, ${ }^{14}$ as well as to intra-atrial conduction delays (IADS). $\mathrm{CO}$ is affected by the epicardial pacing site. ${ }^{12,15,16} \mathrm{BiVP}$ is effective with epicardial leads. ${ }^{17,18}$ BiVP might be effective in either right bundle branch block or left bundle branch block, requiring protocols specific to the conduction block. ${ }^{19}$ Epicardial BiVP might increase arterial systolic pressure and reduce mitral regurgitation. ${ }^{18}$ BiVP might facilitate weaning of critically ill patients from cardiopulmonary bypass. ${ }^{20}$ POPT and BiVP can improve hemodynamics after operations for congenital heart disease. $^{21-23}$ Benefits of BiVP after coronary artery bypass grafting in patients with poor left ventricular function are uncertain. ${ }^{24}$ In experimental animals epicardial DDD pacing reduces $\mathrm{MVO}_{2}$ compared with VVI pacing. ${ }^{11}$ In human subjects endocardial BiVP can improve ventricular function and reduce $\mathrm{MVO}_{2}$, whereas inotropic agents increase $\mathrm{MVO}_{2}{ }^{6}$

\section{Pacing Protocols}

TPCP at physiologic atrial rates typically involves temporary bipolar right atrial and right ventricular wires and a DDD temporary pacemaker. VPS is the anterior or diaphragmatic RV. HR is usually $25 \%$ higher than physiologic rate, and AVD is physiologic. Representative values in adults are HR of 90 beats/min and AVD of $150 \mathrm{~ms}$.

\section{Heart Rate}

The determinants of left ventricular SV are preload (left ventricular end-diastolic volume), afterload (reflecting outflow resistance and ventricular dimensions), and contractile state. Under physiologic conditions, considerable cardiac reserve exists. During exercise, increased venous return can increase preload, $\mathrm{CO}$, and SV. However, cardiac reserve is often diminished after cardiac surgery. Treatment of postoperative low output states includes optimization of preload (with volume administration), afterload (with vasodilators), and contractility (with inotrope administration). Under these circumstances, HR affects systolic or mean arterial pressure (MAP) through several mechanisms. Theoretically, $C O=$ $M A P / R$, where $\mathrm{R}$ is arterial resistance. Thus increases in $\mathrm{CO}$ and MAP are linearly related until $\mathrm{R}$ is affected by reflex changes (Prasso and colleagues, manuscript in preparation). On the other hand, systolic pressure reflects both MAP and pulse pressure, and pulse pressure is affected by SV. If HR is cut in half and $\mathrm{CO}$ remains constant, $\mathrm{SV}$ must double, and systolic pressure will increase. For this reason, MAP is more reliable than systolic blood pressure in POPT.

\section{Atrioventricular Delay}

DDD protocols optimize ventricular filling by synchronizing the onset of ventricular systole with completion of the atrial kick. For patients with regular atrial rhythm, AVD begins with right atrial depolarization or stimulation and ends when the RV depolarizes or is stimulated. The AVD in a permanent pacemaker is typically $150 \mathrm{~ms}$ during atrial sensing and $200 \mathrm{~ms}$ during atrial pacing. The differential of $50 \mathrm{~ms}$ allows for IAD, the interval between the atrial pacing stimulus and the $\mathrm{P}$ wave. IADs are prolonged to 150 to 200 $\mathrm{ms}$ in some patients. A prolonged IAD of $200 \mathrm{~ms}$ combined with an AVD of $200 \mathrm{~ms}$ will result in simultaneous right atrial and right ventricular contraction, negating the atrial kick. Variable IAD and variable conduction time from the RA to the left atrium and atrioventricular node to the RV and the LV require AVD optimization.

\section{Recommendations}

POPT begins with $\mathrm{CO}$ measurement. Many techniques are available for this, but real-time SV by UFP best facilitates POPT. On the downside, application and removal of UFPs requires some manipulation of the ascending aorta. Fluid immersion of the UFP and aorta maximizes ultrasound transmission and data reliability. CO varies with respiration; averaging data over one complete respiratory cycle is most meaningful. Alternative methods for measuring changes in $\mathrm{CO}$ include arterial pulse contour systems (Berberian and coworkers, manuscript in preparation), thermal or indicator dilution, ventricular conductance, echo-Doppler, Fick determinations, ${ }^{22,23}$ or mixed venous oxygen saturation in the RA. Changes in arterial blood pressure can be useful; MAP is most closely related to $\mathrm{CO}$ (Prasso and colleagues, manuscript in preparation).

During TPCP for sinus bradycardia or heart block, POPT involves simply maximizing CO. HR is usually determined by means of clinical assessment of benefits of HR-dependent increases in $\mathrm{CO}$ versus detrimental effects of decreased diastolic filling time and possible subendocardial ischemia. Converting VPS from the RV to a biventricular state can increase $\mathrm{CO}$ in this setting. Temporary epicardial wires can be added to the obtuse margin of the LV. After identifying which lead of the pair has the lowest threshold, BiVP is implemented by bringing the best right ventricular and left ventricular leads to the negative terminal of a temporary pacemaker; the other pair is combined at the positive terminal. If the patient's body is used as the ground, the myocardial wires should be combined at the negative terminal, and the patient's body should be connected to the positive terminal. The output of the temporary pacemaker should be at least twice the value of the right ventricular or 
left ventricular threshold, whichever is higher. Paired temporary atrial wires should be connected as usual. We find these maneuvers consistently increase $\mathrm{CO}$ by $10 \%$ or more in patients with physiologic atrial rates after valve replacement surgery. In some patients, the improvement is $20 \%$ to $30 \%$. BiVP effects on $\mathrm{CO}$ can be quickly tested and a clinical decision reached on whether continuation is warranted, considering a small risk of bleeding on removal of the left ventricular wires. If the benefit does not warrant the risk, the left ventricular wires can be removed. The improvement in $\mathrm{CO}$ with $\mathrm{BiVP}$ is so consistent that it might reflect reversal of the detrimental effects of single-site right ventricular pacing in most instances.

The relationship between AVD and $\mathrm{CO}$ is curvilinear, with CO decreasing at AVDs shorter than $100 \mathrm{~ms}$ or longer than $200 \mathrm{~ms}$. Exceptions are common in advanced heart failure, and objective testing can overcome errors of conventional recommendations. With a long IAD, very long AVDs might be needed for POPT.

RLD is not currently available in temporary pacemakers but is available in some FDA-approved BiVP generators. If permanent BiVP is clinically indicated, it can be implemented with epicardial leads at the conclusion of cardiopulmonary bypass. If this is done, the optimization sequence should be AVD first and then RLD at the optimum AVD. An RLD that results in right ventricular stimulation first is most likely to benefit patients with primary right ventricular dysfunction, ${ }^{25-27}$ whereas the LV first should be favored in left ventricular dysfunction. At present, no reliable guidelines for POPT after cardiac surgery have been developed. The stability of POPT over time is uncertain and could require frequent cycles of measurement and adjustment.

\section{Pacing for Heart Failure}

BiVP might prove valuable in patients undergoing cardiac surgery with advanced preoperative ventricular dysfunction and an IVCD. Little is known about the risks and benefits of pacing for heart failure after cardiac surgery in patients with no other indication. The importance of low EF and IVCD in this setting are largely unproved. Early studies demonstrate beneficial effects of multisite pacing in congenital heart disease with right bundle branch block. ${ }^{22,23}$ Until more information and FDA-approved devices are available, studies of BiVP are best conducted with institutional review board protocols and informed consent.

\section{References}

1. Spotnitz HM. Surgical considerations of pacemakers and automatic defibrillators. In: Cohn L, Edmunds LH Jr, editors. Cardiac surgery in the adult. 2nd ed. New York: McGraw-Hill; 2003. p. 1293-326.

2. Dean DA, JIA C-X, Cabreriza SE, et al. Validation study of a new transit time ultrasonic flow probe for continuous great vessel measurements. ASAIO J. 1996;42:671-6.

3. Leclercq C, Kass DA. Retiming the failing heart: principles and current clinical status of cardiac resynchronization. J Am Coll Cardiol. 2002;39(2):194-201.
4. Abraham WT, Fisher WG, Smith AL, et al. Multicenter InSync Randomized Clinical Evaluation. Cardiac resynchronization in chronic heart failure. $N$ Engl J Med. 2002;346(24):1845-53.

5. Cazeau S, Leclerq C, Lavergne T, et al. Effects of multisite biventricular pacing in patients with heart failure and intraventricular conduction delay. $N$ Engl J Med. 2001;344:873-80.

6. Nelson GS, Berger RD, Fetics BJ, Talbot M, Spinelli JC, Hare JM, et al. Left ventricular or biventricular pacing improves cardiac function at diminished energy cost in patients with dilated cardiomyopathy and left bundle-branch block. Circulation. 2000;102:3053-9.

7. Nelson GS, Curry CW, Wyman BT, et al. Predictors of systolic augmentation from left ventricular preexcitation in patients with dilated cardiomyopathy and intraventricular conduction delay. Circulation. 2000;101(23):2703-9.

8. Kass DA, Chen CH, Curry C, et al. Improved left ventricular mechanics from acute VDD pacing in patients with dilated cardiomyopathy and ventricular conduction delay. Circulation. 1999;99(12):1567-73.

9. Leon AR, Greenberg JM, Kanuru N, et al. Cardiac resynchronization in patients with congestive heart failure and chronic atrial fibrillation: effect of upgrading to biventricular pacing after chronic right ventricular pacing. J Am Coll Cardiol. 2002;39(8):1258-63.

10. Linde C, Leclercq C, Rex S, et al. Long-term benefits of biventricular pacing in congestive heart failure: results from the MUltisite STimulation in cardiomyopathy (MUSTIC) study. J Am Coll Cardiol. 2002; 40(1):111-8.

11. Baller D, Hoeft A, Korb H, Wolpers HG, Zipfel J, Hellige G. Basic physiological studies on cardiac pacing with special reference to the optimal mode and rate after cardiac surgery. Thorac Cardiovasc Surg. 1981;29(3):168-73.

12. Raichlen JS, Campbell FW, Edie RN, Josephson ME, Harken AH. The effect of the site of placement of temporary epicardial pacemakers on ventricular function in patients undergoing cardiac surgery. Circulation. 1984;70(suppl):I118-23.

13. Durbin CG Jr, Kopel RF. Optimal atrioventricular (AV) pacing interval during temporary $\mathrm{AV}$ sequential pacing after cardiac surgery. J Cardiothorac Vasc Anesth. 1993;7(3):316-20.

14. Wish M, Fletcher RD, Gottdiener JS, Cohen AI. Importance of left atrial timing in the programming of dual-chamber pacemakers. Am J Cardiol. 1987;60(7):566-71.

15. Hettrick DA, Euler DE, Pagel PS, et al. Atrial pacing lead location alters the effects of atrioventricular delay on atrial and ventricular hemodynamics. Pacing Clin Electrophysiol. 2002;25(6):888-96.

16. Ansalone G, Giannantoni P, Ricci R, Trambaiolo P, Fedele F, Santini M. Doppler myocardial imaging to evaluate the effectiveness of pacing sites in patients receiving biventricular pacing. J Am Coll Cardiol. 2002;39(3):489-99.

17. Foster AH, Gold MR, McLaughlin JS. Acute hemodynamic effects of atrio-biventricular pacing in humans. Ann Thorac Surg. 1995;59(2): 294-300

18. Tanaka H, Okishige K, Mizuno T, et al. Temporary and permanent biventricular pacing via left ventricular epicardial leads implanted during primary cardiac surgery. Jpn J Thorac Cardiovasc Surg. 2002; 50(7):284-9.

19. Weisse U, Isgro F, Werling Ch, Lehmann A, Saggau W. Impact of atrio-biventricular pacing to poor left-ventricular function after CABG. Thorac Cardiovasc Surg. 2002;50(3):131-5.

20. Kleine P, Doss M, Aybek T, Wimmer-Greinecker G, Moritz A. Biventricular pacing for weaning from extracorporeal circulation in heart failure. Ann Thorac Surg. 2002;73(3):960-2.

21. Janousek J, Vojtovic P, Chaloupecky V, et al. Hemodynamically optimized temporary cardiac pacing after surgery for congenital heart defects. Pacing Clin Electrophysiol. 2000;23(8):1250-9.

22. Zimmerman FJ, Starr JP, Koenig PR, Smith P, Hijazi ZM, Bacha EA. Acute hemodynamic benefit of multisite ventricular pacing after congenital heart surgery. Ann Thorac Surg. 2003;75(6):1775-80.

23. Bacha EA, Zimmerman FJ, Mor-Avi V, et al. Ventricular resynchronization by multisite pacing improves myocardial performance in the postoperative single-ventricle patient. Ann Thorac Surg. 2004;78: 1678-83.

24. Liebold A, Rodig G, Merk J, Birnbaum DE. Short atrioventricular delay dual-chamber pacing early after coronary artery bypass grafting 
in patients with poor left ventricular function. $J$ Cardiothorac Vasc Anesth. 1998;12(3):284-7.

25. Rabkin DG, Cabreriza SE, Curtis LJ, et al. Load dependence of cardiac output in biventricular pacing: right ventricular pressure overload in pigs. J Thorac Cardiovasc Surg. In press.

26. Rabkin DG, Cabreriza SE, Curtis LJ, et al. Mechanisms of optimized biventricular pacing in pulmonary stenosis: effects on left ventricular geometry in swine. Pacing Clin Electrophysiol. 2004;27:1060-71.

27. Rabkin DG, Cabreriza SE, Curtis LJ, Weinberg AD, Hordof AJ, Spotnitz HM. Load dependence of cardiac output in biventricular pacing: right ventricular volume overload in pigs. J Thorac Cardiovasc Surg. In Press.

\section{The Journal of Thoracic and Cardiovascular Surgery Conflict of Interest Policy}

To assure fairness to authors submitting work for consideration in The Journal of Thoracic and Cardiovascular Surgery, a mechanism exists for managing conflicts of interest. The editor and each of the section editors complete a "Conflict of Interest" form that identifies any and all relationships with commercial and other academic entities. When the editor has a potential conflict because of a relationship with another entity or author, the editor appoints an alternate editor from among the section editors or editorial board members who assumes the entire responsibility for final decisions on the manuscript in question. The editor does not read the reviews that are submitted nor engage in discussing the manuscript prior to the final decision. When the conflict of interest involves a section editor, a "guest section editor" is appointed who fills the role normally played by the conflicted section editor. All members of the editorial board and reviewers are asked to indicate any conflict of interest when they agree to review a manuscript. 